\title{
miR-696 plays a role in hepatic gluconeogenesis in ob/ob mice by targeting PGC-1 $\alpha$
}

\author{
ZHIJUAN FANG ${ }^{1}$, PENG LI $^{1}$, WENHUI JIA ${ }^{1}$, TING JIANG ${ }^{1}$, ZHONGYUN WANG $^{2}$ and YANG XIANG ${ }^{1}$ \\ ${ }^{1}$ State Key Laboratory of Pharmaceutical Biotechnology, School of Life Sciences, Nanjing University, Nanjing, \\ Jiangsu 210093; ${ }^{2}$ Department of Anesthesiology, The First Affiliated Hospital, Nanjing Medical University, \\ Nanjing, Jiangsu 210029, P.R. China
}

Received November 10, 2015; Accepted June 14, 2016

DOI: $10.3892 /$ ijmm.2016.2659

\begin{abstract}
MicroRNAs (miRNAs or miRs) are known to play a vital role in type 2 diabetes, and peroxisome proliferatoractivated receptor- $\gamma$ (PPAR $\gamma)$ coactivator- $1 \alpha(\mathrm{PGC}-1 \alpha)$ is involved in the pathogenesis of hepatic insulin resistance. However, the correlation, if any, between PGC-1 $\alpha$ and miRNAs in the disease has not yet been determined. Thus, in the present study, we aimed to examine the correlation between PGC- $1 \alpha$ and miRNAs in diabetes. For this purpose, we used primary hepatocytes isolated from C57BL/6 mice and ob/ob mice. First, we found an inverse correlation between miR-696 and PGC-1 $\alpha$ protein levels in vivo. Second, in vitro evidence demonstrated that PGC-1 $\alpha$ expression was significantly decreased by infection with pre-miR-696-LV, whereas infection with anti-miR-696-LV increased the PGC-1 $\alpha$ protein levels. Third, a luciferase reporter assay confirmed that miR-696 directly recognizes a specific location within the 3'-untranslated region of PGC-1 $\alpha$ transcripts. Furthermore, the biological consequences of miR-696 targeting PGC-1 $\alpha$ were determined by measuring the expression levels of the characteristic hepatic gluconeogenic enzyme, PEPCK, which is regulated by PGC-1 $\alpha$ in the liver via the coactivation of transcription factors. Taken together, our findings demonstrate that miR-696 plays an important role in the development of
\end{abstract}

Correspondence to: Professor Yang Xiang, State Key Laboratory of Pharmaceutical Biotechnology, School of Life Sciences, Nanjing University, 22 Hankou Road, Nanjing, Jiangsu 210093, P.R. China E-mail: xiangy@nju.edu.cn

Dr Zhongyun Wang, Department of Anesthesiology, The First Affiliated Hospital of Nanjing Medical University, 300 Guangzhou Road, Nanjing, Jiangsu 210029, P.R. China

E-mail: zywang1970@126.com

Abbreviations: T2D, type 2 diabetes; IR, insulin resistance; PPAR $\gamma$, peroxisome proliferator-activated receptor $\gamma$; PGC-1 $\alpha$, PPAR $\gamma$ coactivator-1 $\alpha$; miRNAs or miRs, microRNAs; HNF- $4 \alpha$, hepatocyte nuclear factor-4 $\alpha$; FOXO1, forkhead boxO1; PEPCK, phosphoenolpyruvate carboxykinase; G6Pase, glucose-6-phosphatase; RT-qPCR, reverse transcription-quantitative PCR; GFP, green fluorescent protein

Key words: miR-696, peroxisome proliferator-activated receptor $\gamma$ coactivator-1 $\alpha$, hepatic gluconeogenesis, insulin resistance hepatic gluconeogenesis and insulin resistance through the inhibition of PGC-1 $\alpha$ translation in the liver.

\section{Introduction}

Type 2 diabetes (T2D) is a serious chronic metabolic disorder of the 21st century and the fifth leading cause of mortality worldwide $(1,2)$. It is characterized by fasting hyperglycemia, secondary to hepatic insulin resistance (IR) and increased glucose production (3). IR, a main characteristic of T2D, is a pathological state in which insulin function is impaired in target tissues, including the liver, skeletal muscle and adipose tissue. Hepatic IR is of critical importance, as it is the primary disturbance responsible for fasting hyperglycemia (4). Although a number of molecular mechanisms have been suggested to account for the development of hepatic IR $(5,6)$, the underlying mechanism remain unclear.

Peroxisome proliferator-activated receptor- $\gamma$ (PPAR $\gamma$ ) coactivator-1 $\alpha$ (PGC-1 $\alpha$ ), as a nuclear transcriptional coactivator, regulates several important metabolic processes, including mitochondrial biogenesis, adaptive thermogenesis, respiration, insulin secretion and gluconeogenesis $(7,8)$. Previous studies performed in our laboratory, as well as others have demonstrated that PGC-1 $\alpha$ plays an important role in preventing the onset and development of atherosclerosis, as well as in diminishing neointimal formation following balloon vascular injury (9-12). Furthermore, PGC-1 $\alpha$ has been shown to be involved in the pathogenesis of hepatic IR $(3,13)$. Gluconeogenesis is regulated by PGC- $1 \alpha$ in the liver via the coactivation of transcription factors, including hepatocyte nuclear factor- $4 \alpha(\mathrm{HNF}-4 \alpha)$, forkhead boxO1 (FOXO1) and the glucocorticoid receptor, to coordinate the expression of phosphoenolpyruvate carboxykinase (PEPCK) and glucose-6-phosphatase (G6Pase) $(14,15)$. Due to the important role of PGC-1 $\alpha$ in energy metabolism and insulin sensitivity, it seems to be a candidate factor in the etiology of T2D and a drug target for pharmacological intervention (7).

MicroRNAs (miRNAs or miRs) are endogenous non-coding RNA molecules of 19 to 24 nucleotides in length. They play critical roles in the negative regulation of gene expression by base pairing to complementary sites on target mRNAs and then causing a block in translation or triggering the degradation of the target mRNAs (16). It has been has systematically discovered by our group that miRNAs are stably present in human 
serum/plasma and that their unique expression patterns serve as 'fingerprints' of various diseases (17-20). Studies have demonstrated that miRNAs play a vital role in the development of IR, a key pathophysiological link between obesity and diabetes. For instance, the pancreatic islet-specific miR-375 was found to regulate insulin secretion (21). miR-103/107 have been reported to play an important role in insulin sensitivity (22). Ling et al found that miR-320 was able to regulate IR (23). Moreover, muscle IR has been reported to be related to coordinated changes in multiple miRNAs, which indicates that miRNA detection represents a novel molecular biomarker strategy for IR (24).

Recently, miR-696 has been shown to play an important role in regulating PGC-1 $\alpha$ in mouse skeletal muscle in response to physical activity (25). In the present study, we found that hepatic PGC-1 $\alpha$ expression was elevated in ob/ob mice, an animal model of insulin function deficiency, compared with normal C57BL/6 mice. On the contrary, miR-696 expression was significantly lower in the livers of the ob/ob mice compared with the C57BL/6 mice. Hence, we hypothesized that miR-696 may be involved in the function of PGC-1 $\alpha$ in the liver. The direct inhibition of PGC-1 $\alpha$ translation by miR-696 and the potential role of miR-696 in hepatic gluconeogenesis were experimentally validated.

\section{Materials and methods}

Ethics statement and animal models. This study was carried out in the strict accordance with the recommendations in the Guide for the Care and Use of Laboratory Animals of the the National Institutes of Health. The protocol was approved by the Institutional Review Board of Nanjing University, Nanjing, China. Male 8-week-old C57BL/6 and ob/ob mice were obtained from the Model Animal Research Center of Nanjing University. The mice were sacrificed by cervical dislocation, and the livers were removed, immediately frozen in liquid nitrogen, and stored at $-80^{\circ} \mathrm{C}$ for later use.

Cell culture. Primary hepatocytes were isolated from the 8-week-old male C57BL/6 mice by non-recirculating collagenase perfusion, as previously described by Klaunig et al (26). In brief, the livers were initially perfused with $\mathrm{Ca}^{2+}$-free Hank's buffer and then with collagenase type IV in Hank's buffer plus $5 \mathrm{mmol} / 1 \mathrm{CaCl}_{2}$. The dispersed cells were resuspended and seeded onto collagen-coated plates in DMEM supplemented with $10 \%$ fetal bovine serum (FBS) in the presence of penicillin $(100 \mathrm{U} / \mathrm{ml})$, streptomycin $(100 \mathrm{mg} / \mathrm{ml}), 1 \mathrm{mM}$ sodium pyruvate, $1 \mathrm{M}$ dexamethasone and $50 \mathrm{nM}$ insulin. The cultures were maintained at $37^{\circ} \mathrm{C}$ in a humidified atmosphere of $95 \%$ air and $5 \% \mathrm{CO}_{2}$ and were incubated for $12 \mathrm{~h}$ prior to the experiment. The 293T cell line was purchased from the China Cell Culture Center. The 293T cells were cultured in high-glucose DMEM medium (HyClone, Logan, UT, USA) supplemented with $10 \%$ FBS (Gibco, Carlsbad, CA, USA) at $37^{\circ} \mathrm{C}$ in a humidified atmosphere of $95 \%$ air and $5 \% \mathrm{CO}_{2}$.

Infection with lentivirus. Recombinant lentiviruses, respectively carrying pre-mmu-miR-696 precursor (pre-miR-696-LV), pre-non-coding sequence (pre-NC-LV), anti-mmu-miR-696 inhibitor (anti-miR-696-LV) or anti-non-coding sequence (antiNC-LV), were obtained from Hanbio (Shanghai, China).
Each lentivirus contained a green fluorescent protein (GFP) sequence so that the infection efficiency could be monitored by fluorescence. They were individually added to hepatocytes at $30 \%$ confluence in 6 -well plates or $10-\mathrm{cm}$ dishes at an MOI of 40 together with polybrene at a final concentration of $5 \mu \mathrm{g}$ / $\mathrm{ml}$. The cells were then harvested at 3 days post-infection for western blot analysis and reverse transcription-quantitative PCR (RT-qPCR).

Plasmid constructs and luciferase reporter assay. The mouse PGC-1 $\alpha$ 3'-untranslated region (3'-UTR) sequence (1478 bp), obtained from the GenBank database, was amplified by PCR using a mouse genomic DNA template. The PCR products were inserted into the XhoI/NotI sites of the psiCHECK-2 plasmid (Promega, Madison, WI, USA) using the following primers: forward, 5'-GCGCTCGAGGAGATGGTCAATACC TCATGGG-3' and reverse, 5'-AATGCGGCCGCAGAAAAT GTGGAAAAATATTGC-3'. Efficient insertion was confirmed by sequencing. For the luciferase reporter assays, the $293 \mathrm{~T}$ cells were cultured in 6-well plates. For each well, the cells at $70-80 \%$ confluence were co-transfected with $2 \mu \mathrm{g}$ of the psiCHECK-2-PGC-1 $\alpha$ 3'-UTR plasmid and $2 \mu \mathrm{g}$ of the pHBLV-pre-miR-696, pHBLV-anti-miR-696 or pHBLV-IRES zsgreen plasmid using Lipofectamine 2000 (Invitrogen, Grand Island, NY, USA) according to the manufacturer's instructions. The cells were assayed using luciferase assay kits (Promega) at $24 \mathrm{~h}$ post-transfection.

$R N A$ isolation and $R T-q P C R$. Total RNA derived from the cells and liver tissues was extracted using TRIzol reagent (Invitrogen, Carlsbad, CA, USA) according to the manufacturer's instructions. RT-qPCR was carried out using TaqMan miRNA probes (Applied Biosystems, Foster City, CA, USA) for miRNAs and specific primers for mRNA detection, respectively according to the manufacturer's instructions. Briefly, for miRNAs, $1 \mu \mathrm{g}$ of total RNA was reverse transcribed into cDNA using AMV reverse transcriptase (Takara, Dalian, China) and stem-loop RT primers (Applied Biosystems). Quantitative (qPCR) was performed using a TaqMan PCR kit on an Applied Biosystems 7300 Sequence Detection system (Applied Biosystems); for mRNAs, $1 \mu \mathrm{g}$ of RNA was reverse transcribed into cDNA with oligo $(\mathrm{dT})_{18}$ primers (Takara) and AMV reverse transcriptase at $42^{\circ} \mathrm{C}$ for $1 \mathrm{~h}$, followed by $70^{\circ} \mathrm{C}$ for $10 \mathrm{~min}$. RT-qPCR was performed using the Applied Biosystems 7300 Sequence Detection system with forward and reverse primers for specific genes synthesized by Invitrogen, as well as EvaGreen (Takara); the amplification conditions were as follows: 1 cycle of $95^{\circ} \mathrm{C}$ for $5 \mathrm{~min}$ followed by 40 cycles of $95^{\circ} \mathrm{C}$ for $30 \mathrm{sec}, 60^{\circ} \mathrm{C}$ for $30 \mathrm{sec}$, and final 1 cycle of $72^{\circ} \mathrm{C}$ for $30 \mathrm{sec}$. The primers sequences used in this study are listed in Table I. The primers specific for miR-696 cannot be disclosed (Applied Biosystems). All reactions were run in triplicate. After the reactions were complete, the $\mathrm{C}_{\mathrm{T}}$ values were determined using fixed threshold settings. miRNA expression was normalized to U6 snRNA expression in this study. The amount of miRNA to relative to the internal control U6 was calculated using the equation $2^{-\Delta \mathrm{CT}}$, in which $\Delta \mathrm{C}_{\mathrm{T}}=\mathrm{C}_{\mathrm{T}}$ miRNA - CT U6.

Western blot analysis. To obtain total proteins, liver tissues from the mice or the cells (hepatocytes) were lysed in a buffer 
Table I. PCR primers used in this study.

mRNA

Primer sequences

Mouse U6 snRNA

Sense: 5'-CGGGATCCGATCCGACGCCGCCATCTCTAG-3'

Mouse PGC-1 $\alpha$

Antisense: 5'-CGGTCGACTAGTATATGTGCTGCCGAAGCG-3'

Sense: $\quad$ 5'-GCGCTCGAGGAGATGGTCAATACCTCATGGG-3'

Antisense: 5'-AATGCGGCCGCAGAAAATGTGGAAAAATATTGC-3'

The primers specific for miR-696 cannot be disclosed. PGC-1 $\alpha$, peroxisome proliferator-activated receptor- $\gamma$ coactivator- $1 \alpha$ (PGC-1 $\alpha)$.

A

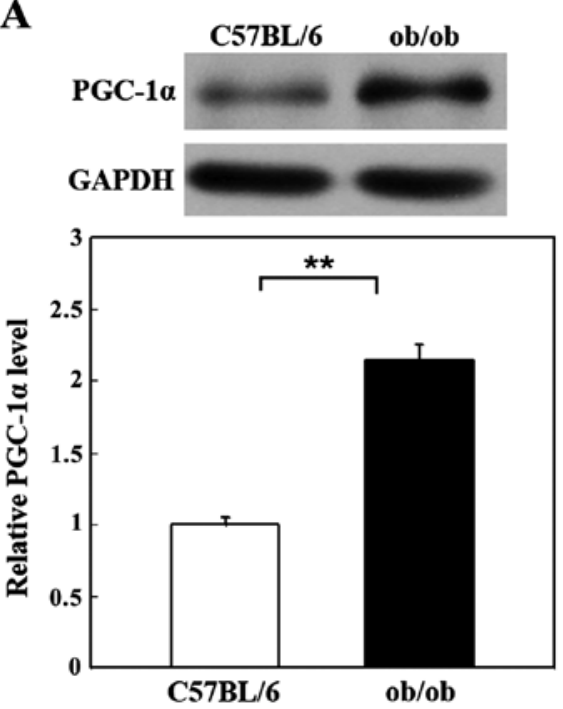

B

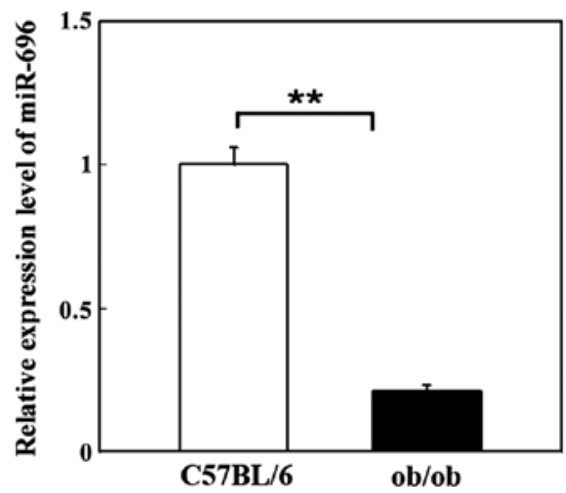

Figure 1. Inverse correlation between the peroxisome proliferator-activated receptor- $\gamma$ coactivator-1 $\alpha$ (PGC-1 $\alpha)$ protein level and miR-696 expression in mouse liver. (A) Western blot analysis of PGC-1 $\alpha$ protein expression in the livers of ob/ob mice and C57BL/6 mice. Quantification of protein bands was performed using Image software. The data are presented as the means $\pm \mathrm{SE}, \mathrm{n}=5,{ }^{* *} \mathrm{p}<0.01$. (B) Comparison of the miR-696 relative expression level in liver between ob/ob mice and C57BL/6 mice using a hydrolysis probe-based RT-qPCR assay. The data are presented as the means $\pm \mathrm{SE}, \mathrm{n}=10^{* *} \mathrm{p}<0.01$.

containing $50 \mathrm{mM}$ Tris- $\mathrm{HCl}$ (pH 7.4), $150 \mathrm{mM} \mathrm{NaCl}, 1 \%$ Triton $\mathrm{X}-100,0.1 \%$ SDS, and $1 \mathrm{mM}$ PMSF. The cell lysates were then centrifuged at $13,000 \times \mathrm{g}$ for $5 \mathrm{~min}$ at $4^{\circ} \mathrm{C}$ and supernatants were collected for western blot analysis. We determined the protein concentrations using the BCA method (Pierce, Rockford, IL, USA). Equal amounts of protein $(40 \mu \mathrm{g})$ were separated by $10 \%$ SDS-PAGE and transferred onto PVDF membranes. The membranes were blocked for $1 \mathrm{~h}$ with $5 \%$ non-fat milk in TBST, followed by overnight incubation at $4^{\circ} \mathrm{C}$ with

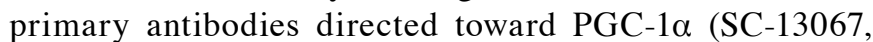
1:1000) and PEPCK (SC-271029, 1:1000) (all from Santa Cruz Biotechnology, Santa Cruz, CA, USA). After washing, the membranes were incubated with corresponding secondary antibody conjugated to horseradish peroxidase. Normalization was performed by blotting the same samples with an antibody against GAPDH (Santa Cruz Biotechnology).

Statistical analyses. All images of western blot analyses are representative of at least 3 independent experiments. For each experiment, RT-qPCR assays were performed in triplicate. The data shown are presented as the means \pm standard error for 3 independent experiments. Differences were considered statistically significant at $\mathrm{P}<0.05$ and were assessed using the Student's t-test.

\section{Results}

Evaluation of PGC-1 $\alpha$ and miR-696 expression in the livers of $o b / o b$ mouse. In order to investigate the biological function of PGC-1 $\alpha$ in livers of mice with IR, we examined the protein level of PGC-1 $\alpha$ in a mouse model of genetic IR and obesity (ob/ob mice). C57BL/6 mice of the same age were used as controls. As shown in Fig. 1A, the protein expression level of PGC-1 $\alpha$ was significantly increased in the livers of ob/ob mice (2.15-fold that of the normal C57BL/6 mice). The results are representative data from 5 independent experiments. Quantification of the protein bands was performed using Image software. These results suggest that PGC-1 $\alpha$ is involved in IR in the liver.

Recently, PGC-1 $\alpha$ was deduced to be a target of miR-696 not only by computational prediction, but also by validating an inverse correlation between miR-696 and the PGC- $1 \alpha$ protein level in mouse skeletal muscle (25). In this study, in order to investigate the correlation between miR-696 and PGC- $1 \alpha$ in mouse liver, we examined the level of miR-696 by RT-qPCR in the livers of ob/ob mice and C57BL/6 mice. We found that the miR-696 level in the livers of ob/ob mice was downregulated significantly (only 0.21 -fold that of the normal controls; Fig. 1B). Taken together, these results suggest that miR-696 negatively regulates PGC-1 $\alpha$ expression in mouse liver. 
$\mathbf{A}$
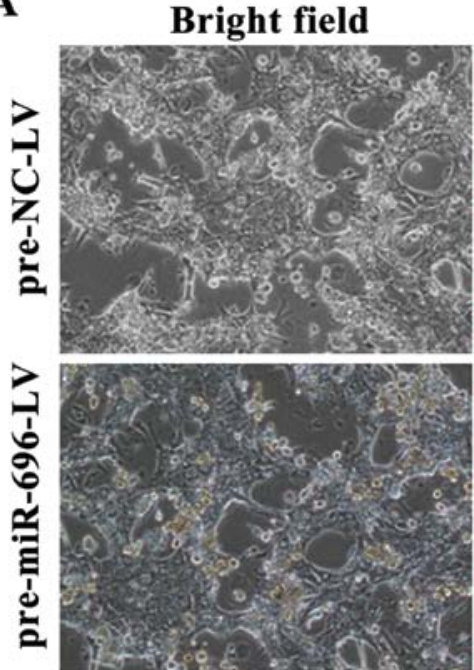

B
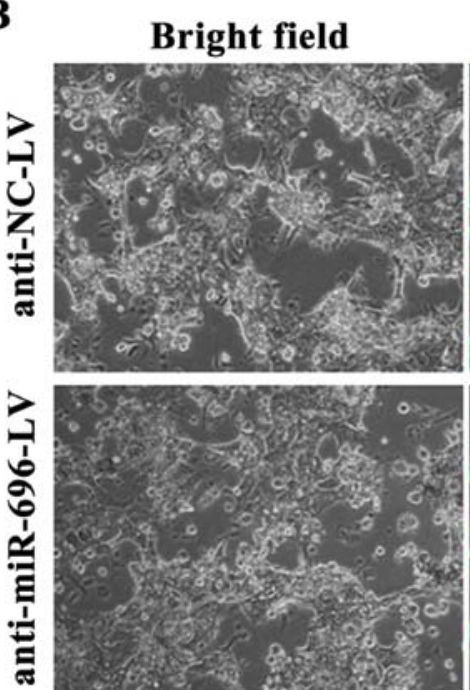

Fluorescence
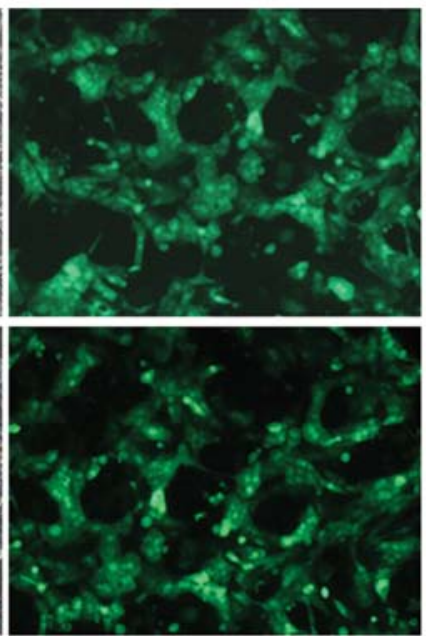

Fluorescence
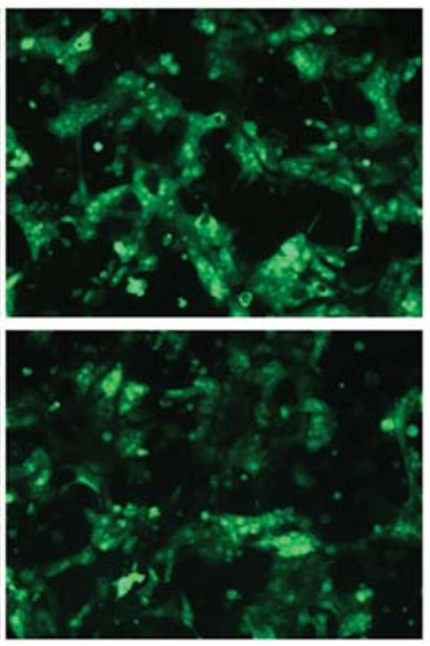

Figure 2. Observation of cells infected with pre-miR-696-LV or anti-miR-696-LV by fluorescence microscopy. (A) Observation of cells infected with premiR-696-LV or pre-NC-LV after $72 \mathrm{~h}$ by fluorescence microscopy. pre-miR-696-LV, lentivirus carrying pre-miR-696 precursor; pre-NC-LV, lentivirus carrying pre-non-coding sequence; (B) Observation of cells infected with anti-miR-696-LV or anti-NC-LV after $72 \mathrm{~h}$ by fluorescence microscopy. anti-miR-696-LV, lentivirus carrying anti-miR-696 inhibitor; anti-NC-LV, lentivirus carrying anti-non-coding sequence.

Regulation of $P G C-1 \alpha$ by miR-696 in primary hepatocytes. We further examined the correlation between miR-696 and PGC-1 $\alpha$ by evaluating the expression of PGC- $1 \alpha$ in primary hepatocytes following the overexpression or knockdown of miR-696. In our study, miR-696 overexpression/knockdown was achieved by infecting mouse primary hepatocytes with pre-miR-696-LV/anti-miR-696-LV. Primary hepatocytes infected by pre-NC-LV or anti-NC-LV in the same manner were used as the controls. The efficiency of infection at $72 \mathrm{~h}$ screened by the GFP signal is shown in Fig. 2. We also measured the efficiency of infection by RT-qPCR. As shown in Fig. 3A, the level of miR-696 was increased 6.7-fold in the primary hepatocytes infected by pre-miR-696-LV compared with the controls. However, the miR-696 level was reduced by $59 \%$ after the primary hepatocytes were infected by the anti-miR-696-LV compared with anti-NC-LV (Fig. 3B). The expression of PGC-1 $\alpha$ was decreased by $41 \%$ in the hepatocytes in which miR-696 was overexpressed, and it was enhanced by $87 \%$ in the hepatocytes in which miR-696 was knocked down (Fig. 3C). These results prove that miR-696 negatively regulates PGC-1 $\alpha$ in primary hepatocytes.

Identification of PGC-1 $\alpha$ as a target of miR-696 by luciferase binding assays. We found that there was perfect base pairing between the 'seeds' (the core sequence that encompasses the first 2-8 bases of the mature miRNA) and cognate targets (Fig. 4A). Thus, we then conducted a luciferase binding assays using a psiCHECK-2 luciferase plasmid to determine whether miR-696 suppresses PGC-1 $\alpha$ through direct binding to its 3'-UTR. As expected, the overexpression of miR-696 resulted in a $62 \%$ reduction of firefly luciferase reporter activity. However, the knockdown of miR-696 resulted in a 17\% increment of firefly luciferase reporter activity (Fig. 4B). These results unequivocally demonstrate that miR-696 directly recognizes the 3'-UTR of the PGC-1 $\alpha$ transcript. Thus, the upregulation of miR-696 promotes the suppression of PGC-1 $\alpha$. 
$\mathbf{A}$

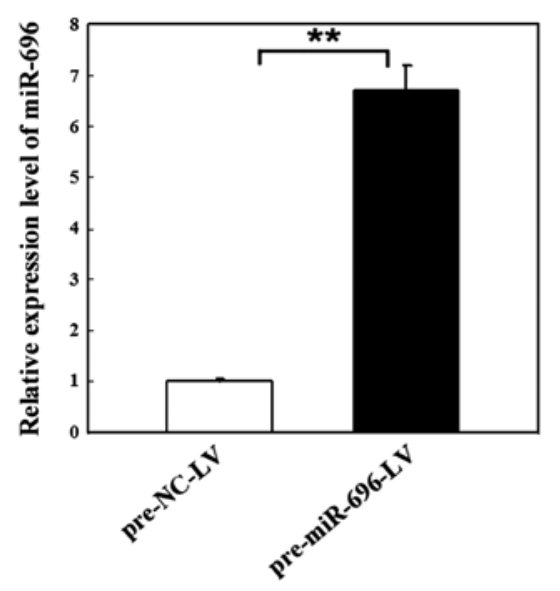

B

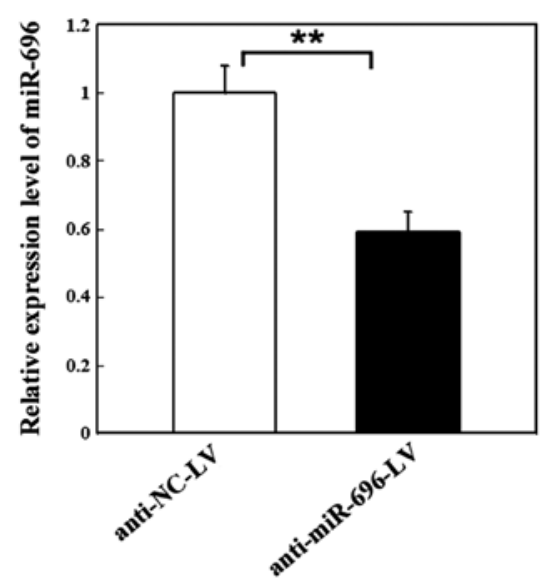

C

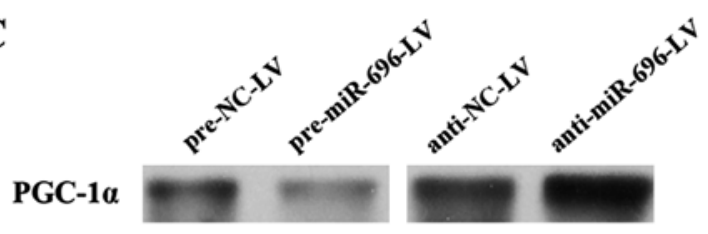

GAPDH

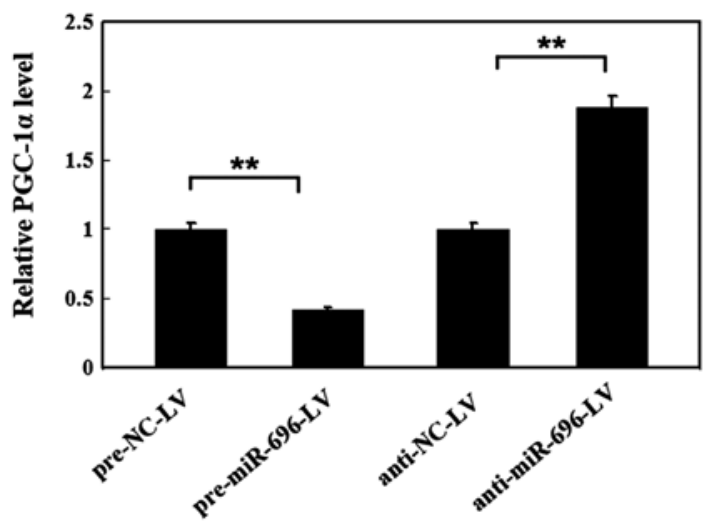

Figure 3. Identification of peroxisome proliferator-activated receptor- $\gamma$ coactivator-1 $\alpha$ (PGC-1 $\alpha$ ) as a target of miR-696 in primary hepatocytes (A and B) Comparison of miR-696 relative expression level in primary hepatocytes following infection with pre-miR-696-LV or anti-miR-696-LV using a hydrolysis probe-based RT-qPCR assay. (C) Western blot analysis of PGC-1 $\alpha$ protein expression in primary hepatocytes following infection with pre-miR-696-LV or anti-miR-696-LV. One representative experiment out of 3 is shown. Quantification of protein bands was performed using Image software. The data are presented as the means $\pm \mathrm{SE}, \mathrm{n}=5,{ }^{* *} \mathrm{p}<0.01$.

A

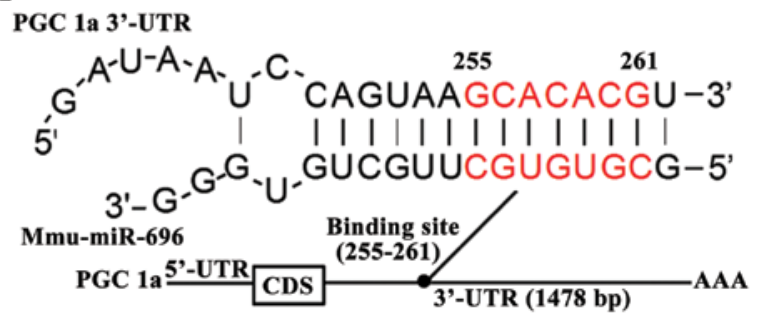

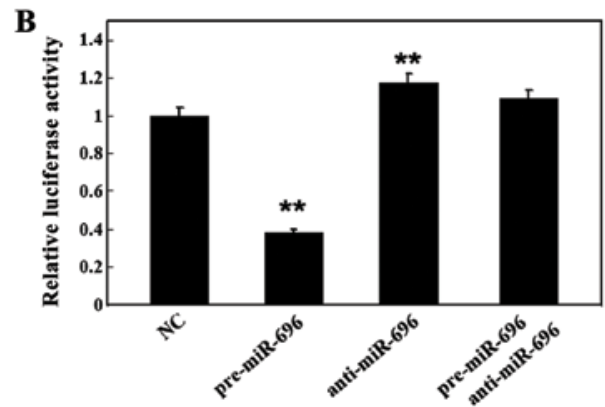

Figure 4. Hybridization of miR-696 and peroxisome proliferator-activated receptor- $\gamma$ coactivator-1 $\alpha$ (PGC-1 $\alpha$ ), and relative luciferase activity. (A) Schematic depicting the conserved binding sites for miR-696. The seed-recognizing site is marked in red; all nucleotides in this region were completely conserved. Hypothesized duplexes formed by the interaction of the binding sites of PGC-1 $\alpha$ 3'-untranslated region (3'-UTR) (top) and miR-696 (bottom) are illustrated. The thick lines between the two chains represent hydrogen bonds between adenine (A)-thymine (T) pairs or guanine (G)-cytosine (C) pairs, while thin lines represent G-U pairings. (B) Relative luciferase activity after transfection with pre-miR-696 or anti-miR-696 in primary hepatocytes. Primary hepatocytes were co-transfected with $2 \mu \mathrm{g}$ of the psicheck-2-PGC-1 $\alpha$ 3'-UTR plasmid and $2 \mu \mathrm{g}$ of pHBLV-pre-miR-696, pHBLV-anti-miR-696 or pHBLV-IRES zsgreen plasmid. The data are presented as the means $\pm \mathrm{SE},{ }^{* *} \mathrm{p}<0.01$. 
A

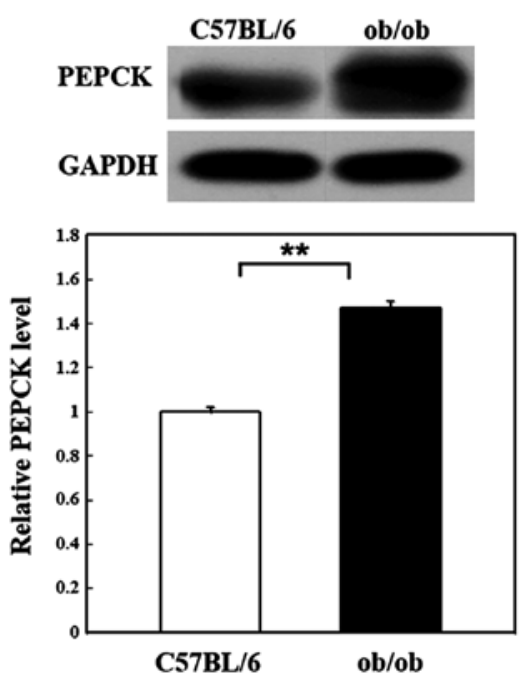

B
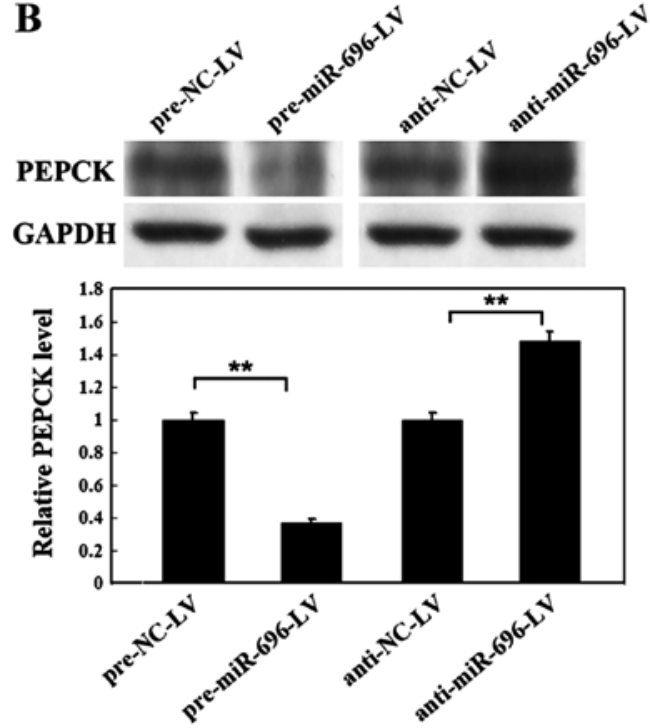

Figure 5. Western blot analysis of phosphoenolpyruvate carboxykinase (PEPCK) protein expression. (A) Western blot analysis of PEPCK protein expression in livers of ob/ob mice and C57BL/6 mice. (B) Western blot analysis of PEPCK protein expression in primary hepatocytes following infection with premiR-696-LV or anti-miR-696-LV. One representative experiment out of 3 is shown. Quantification of protein bands was performed using Image software. The data are presented as the means $\pm \mathrm{SE}, \mathrm{n}=5,{ }^{* *} \mathrm{p}<0.01$.

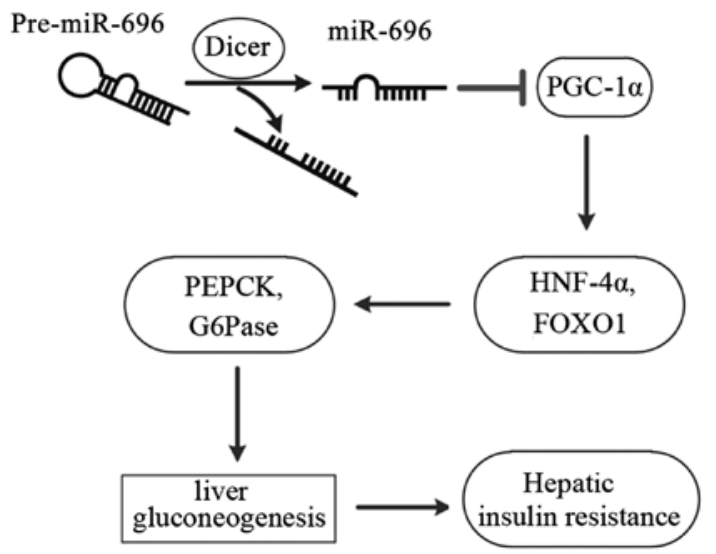

Figure 6. An illustration of the entire signaling pathway described in this study, including the interaction between miR-696 and peroxisome proliferatoractivated receptor- $\gamma$ coactivator- $1 \alpha$ (PGC- $1 \alpha)$, along with a brief description of the pathway mentioned in this study.

Role of miR-696 targeting PGC-1 $\alpha$ in hepatic gluconeogenesis. PGC- $1 \alpha$ has been reported to be an improtant regulator of gluconeogenesis in the liver $(13,14,27)$. It can coordinate the expression of characteristic hepatic gluconeogenic enzymes, including PEPCK and G6Pase through coactivating transcription factors, such as HNF-4 $\alpha$, FOXO1 $(14,15)$. We then examined the expression of PEPCK as an indicator to examine the downstream biological consequences of the miR-696driven inhibition of PGC-1 $\alpha$ expression. As shown in Fig. 5A, the protein level of PEPCK was increased by $47 \%$ in the livers of ob/ob mice compared with the normal C57BL/6 mice. We then analyzed the molecular biological consequences of the miR-696-driven inhibition of PGC-1 $\alpha$ gene expression in mouse primary hepatocytes. At $72 \mathrm{~h}$ following the overexpression or knockdown of miR-696, the expression level of PEPCK was significantly inhibited by infection with pre-miR-696-LV (only
0.37 -fold that of the control). The PEPCK level was significantly elevated by infection with anti-miR-696-LV (1.51-fold that of the control) (Fig. 5B). These downstream effects further support the hypothesis that miR-696 negatively regulates PGC- $1 \alpha$ protein expression in the mouse liver and then coordinates the expression of characteristic hepatic gluconeogenic enzymes, including PEPCK through coactivating transcription factors, such as HNF- $4 \alpha$, FOXO1, and therefore plays an important role in hepatic gluconeogenesis and IR (Fig. 6).

\section{Discussion}

In this study, we identified an inverse correlation between miR-696 and PGC- $1 \alpha$ in vivo and in vitro, and found that PGC- $1 \alpha$ expression was significantly decreased by the overexpression of miR-696 through pre-miR-696-LV, whereas the knockdown of miR-696 increased the protein level of PGC-1 $\alpha$. The results of luciferase reporter assay confirmed that miR-696 directly recognizes a specific location within the 3'-UTR of PGC-1 $\alpha$ transcripts. The molecular biological consequences of the miR-696-driven inhibition of PGC-1 $\alpha$ expression were examined in the livers of ob/ob mice and primary hepatocytes using PEPCK as an indicator. We present convincing evidence that miR-696 plays a significant role in hepatic gluconeogenesis and IR through the inhibition of PGC- $1 \alpha$.

miRNAs are small non-coding transcripts that are widely expressed in plants and animals to regulate gene expression post-transcriptionally by the cleavage or translational repression of their specific target mRNAs (28). It has been indicated by bioinformatic studies that miRNAs have the potential to regulate the expression of a wide spectrum of target genes (29). miRNAs have been reported to play important roles in many diseases, including T2D $(30,31)$. For example, the let-7 family influence insulin sensitivity (32). Moreover, miRNAs such as miR-29, miR-138, miR-143, miR-326 and miR-21 play an important role in regulating glucose metabolism (33-37). 
PGC-1 $\alpha$ is known to be a potent regulator of gluconeogenesis in the liver by coactivating transcription factors, including HNF-4 $\alpha$, FOXO1. Aoi et al (25) recently reported that PGC-1 $\alpha$ is directly regulated by miR-696 in mouse skeletal muscle. Considering these findings, we conducted our experiments with miR-696 to determine its role in hepatic gluconeogenesis and IR via PGC-1 $\alpha$. Our results demonstrated an inverse correlation between miR-696 and PGC-1 $\alpha$ in the livers of ob/ob mice compared with normal C57BL/6 mice. Additionally, an increase in miR-696 expression in primary hepatocytes led to the downregulation of PGC-1 $\alpha$ protein, whereas the knockdown of miR-696 had the opposite effect on PGC-1 $\alpha$ expression. These observations strongly suggest that miR-696 negatively regulates the expression of PGC- $1 \alpha$ in the liver.

The liver plays a significant role in maintaining normal circulatory glucose levels and the gluconeogenic pathway is a predominant contributor towards this phenomenon. PEPCK, as the rate limiting enzyme of gluconeogenesis, catalyzes the conversion of oxaloacetate to phosphoenolpyruvate. Taking into account that the major downstream pathway of insulin function in the liver culminates into the inhibition of gluconeogenic genes, we used PEPCK as an important indicator and determined the effects of miR-696 in hepatic gluconeogenesis on the status of PEPCK. As compared to the control, the protein level of PEPCK was increased by $47 \%$ in the livers of ob/ob mice compared with normal C57BL/6 mice. In primary hepatocytes, we also found that the expression of PEPCK was suppressed by infecting mouse primary hepatocytes with pre-miR-696-LV, and stimulated by infecting mouse primary hepatocytes with anti-miR-696-LV. These results suggest that miR-696 plays a vital role in hepatic gluconeogenesis and IR.

In conclusion, our in vitro and in vivo experiments may help us to understand the importance and mechanisms of action of miR-696 in regulating PGC-1 $\alpha$ in hepatic gluconeogenesis and IR, and provide hope for developing miRNA-targeting agents for preventing and treating IR in T2D.

\section{Acknowledgements}

This study was supported by grants from the National Natural Science Foundation of China (nos. 30570731, 30871195, 81070653, 81270907 and 81370926) to Yang Xiang.

\section{References}

1. Shaw JE, Sicree RA and Zimmet PZ: Global estimates of the prevalence of diabetes for 2010 and 2030. Diabetes Res Clin Pract 87: 4-14, 2010.

2. Olokoba AB, Obateru OA and Olokoba LB: Type 2 diabetes mellitus: a review of current trends. Oman Med J 27: 269-273, 2012.

3. Liang H, Balas B, Tantiwong P, Dube J, Goodpaster BH, O'Doherty RM, DeFronzo RA, Richardson A, Musi N and Ward WF: Whole body overexpression of PGC-1 $\alpha$ has opposite effects on hepatic and muscle insulin sensitivity. Am J Physiol Endocrinol Metab 296: E945-E954, 2009.

4. Kraegen EW, Clark PW, Jenkins AB, Daley EA, Chisholm DJ and Storlien LH: Development of muscle insulin resistance after liver insulin resistance in high-fat-fed rats. Diabetes 40 : 1397-1403, 1991.

5. Bajaj M and Defronzo RA: Metabolic and molecular basis of insulin resistance. J Nucl Cardiol 10: 311-323, 2003.

6. Yamada N: Molecular basis of metabolic syndrome: insulin resistance. Nihon Rinsho 64 (Suppl 9): 23-29, 2006 (In Japanese).
7. Russell AP: PGC-1alpha and exercise: important partners in combating insulin resistance. Curr Diabetes Rev 1: 175-181, 2005.

8. Handschin C and Spiegelman BM: Peroxisome proliferator-activated receptor gamma coactivator 1 coactivators, energy homeostasis, and metabolism. Endocr Rev 27: 728-735, 2006.

9. Xu W, Guo T, Zhang Y, Jiang X, Zhang Y, Zen K, Yu B and Zhang CY: The inhibitory effect of dexamethasone on platelet-derived growth factor-induced vascular smooth muscle cell migration through up-regulating PGC-1 $\alpha$ expression. Exp Cell Res 317: 1083-1092, 2011.

10. Zhang Y, Liu C, Zhu L, Jiang X, Chen X, Qi X, Liang X, Jin S, Zhang P, Li Q, et al: PGC-1alpha inhibits oleic acid induced proliferation and migration of rat vascular smooth muscle cells. PLoS One 2: e1137, 2007.

11. Jiang X, Zhang Y, Hou D, Zhu L, Xu W, Ding L, Qi X, Sun G, Liu C, Zhang J, et al: 17beta-estradiol inhibits oleic acid-induced rat VSMC proliferation and migration by restoring PGC-1alpha expression. Mol Cell Endocrinol 315: 74-80, 2010.

12. Zhu L, Sun G, Zhang H, Zhang Y, Chen X, Jiang X, Jiang X, Krauss S, Zhang J, Xiang Y and Zhang CY: PGC-1alpha is a key regulator of glucose-induced proliferation and migration in vascular smooth muscle cells. PLoS One 4: e4182, 2009.

13. Koo SH, Satoh H, Herzig S, Lee CH, Hedrick S, Kulkarni R, Evans RM, Olefsky J and Montminy M: PGC-1 promotes insulin resistance in liver through PPAR-alpha-dependent induction of TRB-3. Nat Med 10: 530-534, 2004.

14. Puigserver P, Rhee J, Donovan J, Walkey CJ, Yoon JC, Oriente F, Kitamura Y, Altomonte J, Dong H, Accili D and Spiegelman BM: Insulin-regulated hepatic gluconeogenesis through FOXO1-PGC-1 $\alpha$ interaction. Nature 423: 550-555, 2003.

15. Rhee J, Inoue Y, Yoon JC, Puigserver P, Fan M, Gonzalez FJ and Spiegelman BM: Regulation of hepatic fasting response by PPARgamma coactivator-1 $\alpha$ (PGC-1): requirement for hepatocyte nuclear factor $4 \alpha$ in gluconeogenesis. Proc Natl Acad Sci USA 100: 4012-4017, 2003.

16. Griffiths-Jones S, Grocock RJ, van Dongen S, Bateman A and Enright AJ: miRBase: microRNA sequences, targets and gene nomenclature. Nucleic Acids Res 34: D140-D144, 2006.

17. Wang C, Yang C, Chen X, Yao B, Yang C, Zhu C, Li L, Wang J, Li X, Shao Y, et al: Altered profile of seminal plasma microRNAs in the molecular diagnosis of male infertility. Clin Chem 57: 1722-1731, 2011.

18. Liu R, Zhang C, Hu Z, Li G, Wang C, Yang C, Huang D, Chen X, Zhang $\mathrm{H}$, Zhuang $\mathrm{R}$, et al: A five-microRNA signature identified from genome-wide serum microRNA expression profiling serves as a fingerprint for gastric cancer diagnosis. Eur J Cancer 47: 784-791, 2011.

19. Chen X, Ba Y, Ma L, Cai X, Yin Y, Wang K, Guo J, Zhang Y, Chen J, Guo X, et al: Characterization of microRNAs in serum: a novel class of biomarkers for diagnosis of cancer and other diseases. Cell Res 18: 997-1006, 2008.

20. Chen X, Hu Z, Wang W, Ba Y, Ma L, Zhang C, Wang C, Ren Z, Zhao Y, Wu S, et al: Identification of ten serum microRNAs from a genome-wide serum microRNA expression profile as novel noninvasive biomarkers for nonsmall cell lung cancer diagnosis. Int J Cancer 130: 1620-1628, 2012.

21. Fernandez-Valverde SL, Taft RJ and Mattick JS: MicroRNAs in $\beta$-cell biology, insulin resistance, diabetes and its complications. Diabetes 60: 1825-1831, 2011.

22. Trajkovski M, Hausser J, Soutschek J, Bhat B, Akin A, Zavolan M, Heim MH and Stoffel M: MicroRNAs 103 and 107 regulate insulin sensitivity. Nature 474: 649-653, 2011.

23. Ling HY, Ou HS, Feng SD, Zhang XY, Tuo QH, Chen LX, Zhu BY, Gao ZP, Tang CK, Yin WD, et al: CHANGES IN microRNA $(\mathrm{miR})$ profile and effects of miR-320 in insulin-resistant 3T3-L1 adipocytes. Clin Exp Pharmacol Physiol 36: e32-e39, 2009.

24. Gallagher IJ, Scheele C, Keller P, Nielsen AR, Remenyi J, Fischer CP, Roder K, Babraj J, Wahlestedt C, Hutvagner G, et al: Integration of microRNA changes in vivo identifies novel molecular features of muscle insulin resistance in type 2 diabetes. Genome Med 2: 9, 2010.

25. Aoi W, Naito Y, Mizushima K, Takanami Y, Kawai Y, Ichikawa $\mathrm{H}$ and Yoshikawa T: The microRNA miR-696 regulates PGC-1 $\{\alpha\}$ in mouse skeletal muscle in response to physical activity. Am J Physiol Endocrinol Metab 298: E799-E806, 2010.

26. Klaunig JE, Goldblatt PJ, Hinton DE, Lipsky MM and Trump BF: Mouse liver cell culture. II. Primary culture. In Vitro 17: 926-934, 1981. 
27. Yoon JC, Puigserver P, Chen G, Donovan J, Wu Z, Rhee J, Adelmant G, Stafford J, Kahn CR, Granner DK, et al: Control of hepatic gluconeogenesis through the transcriptional coactivator PGC-1. Nature 413: 131-138, 2001.

28. Bartel DP: MicroRNAs: genomics, biogenesis, mechanism, and function. Cell 116: 281-297, 2004.

29. Lewis BP, Burge CB and Bartel DP: Conserved seed pairing, often flanked by adenosines, indicates that thousands of human genes are microRNA targets. Cell 120: 15-20, 2005.

30. Sayed D and Abdellatif M: MicroRNAs in development and disease. Physiol Rev 91: 827-887, 2011.

31. Poy MN, Eliasson L, Krutzfeldt J, Kuwajima S, Ma X, Macdonald PE, Pfeffer S, Tuschl T, Rajewsky N, Rorsman P and Stoffel M: A pancreatic islet-specific microRNA regulates insulin secretion. Nature 432: 226-230, 2004.

32. Frost RJ and Olson EN: Control of glucose homeostasis and insulin sensitivity by the Let-7 family of microRNAs. Proc Natl Acad Sci USA 108: 21075-21080, 2011.

33. He A, Zhu L, Gupta N, Chang Y and Fang F: Overexpression of micro ribonucleic acid 29 , highly up-regulated in diabetic rats, leads to insulin resistance in 3T3-L1 adipocytes. Mol Endocrinol 21: 2785-2794, 2007.
34. Pandey AK, Verma G, Vig S, Srivastava S, Srivastava AK and Datta M: miR-29a levels are elevated in the $\mathrm{db} / \mathrm{db}$ mice liver and its overexpression leads to attenuation of insulin action on PEPCK gene expression in HepG2 cells. Mol Cell Endocrinol 332: 125-133, 2011.

35. Peschiaroli A, Giacobbe A, Formosa A, Markert EK, Bongiorno-Borbone L, Levine AJ, Candi E, D'Alessandro A, Zolla L, Finazzi Agrò A and Melino G: miR-143 regulates hexokinase 2 expression in cancer cells. Oncogene 32: 797-802, 2013.

36. Kefas B, Comeau L, Erdle N, Montgomery E, Amos S and Purow B: Pyruvate kinase M2 is a target of the tumor-suppressive microRNA-326 and regulates the survival of glioma cells. Neuro-oncol 12: 1102-1112, 2010.

37. Vinciguerra M, Sgroi A, Veyrat-Durebex C, Rubbia-Brandt L, Buhler LH and Foti M: Unsaturated fatty acids inhibit the expression of tumor suppressor phosphatase and tensin homolog (PTEN) via microRNA-21 up-regulation in hepatocytes. Hepatology 49: 1176-1184, 2009. 